\title{
Local Radiotherapy or Chemotherapy for Oligo-recurrent Cervical Cancer in Patients With Prior Pelvic Irradiation
}

\author{
KAZUMA KOBAYASHI ${ }^{1,2,3}$, NAOYA MURAKAMI ${ }^{4}$, KANA TAKAHASHI $^{4}$, \\ KOJI INABA $^{4}$, RYUJI HAMAMOTO ${ }^{1,2,3}$ and JUN ITAMI ${ }^{4}$ \\ ${ }^{1}$ Division of Molecular Modification and Cancer Biology, National Cancer Center Research Institute, Tokyo, Japan; \\ ${ }^{2}$ Cancer Translational Research Team, RIKEN Center for Advanced Intelligence Project, Tokyo, Japan; \\ ${ }^{3}$ Department of NCC Cancer Science, Graduate School of Medical and Dental Sciences, \\ Tokyo Medical and Dental University, Tokyo, Japan; \\ ${ }^{4}$ Department of Radiation Oncology, National Cancer Center Hospital, Tokyo, Japan
}

\begin{abstract}
Background/Aim: The aim of this study was to compare the clinical outcomes of patients treated by local radiotherapy or chemotherapy for oligo-recurrent cervical cancer with prior pelvic irradiation. Patients and Methods: Forty-one patients who had received pelvic irradiation for cervical cancer developed oligo-recurrent ( $\leq 5$ lesions) relapses and underwent local radiotherapy $(n=22)$ or systemic chemotherapy $(n=19)$. Overall survival (OS), local recurrence-free survival (LRFS) and distant-free survival (DFS) were estimated, and risk factors were identified. Results: The median follow-up was 24.1 months. The group of local radiotherapy showed a significantly superior LRFS, but inferior DFS. There was no significant difference in OS. Multivariate analysis revealed that FIGO stage at initial diagnosis was associated with OS. For patients with early FIGO stages (IB-IIB), local radiotherapy provided a tendency toward longer OS than chemotherapy. Conclusion: Out-field oligo-recurrence in patients with initial early FIGO stages may be an indication of salvage radiotherapy.
\end{abstract}

Treatment selection of recurrent cervical cancer remains a major therapeutic challenge, especially for cases with prior pelvic irradiation. Five-year loco-regional and distant failure rates are as high as $20-40 \%$ in patients with International

This article is freely accessible online.

Correspondence to: Kazuma Kobayashi, MD, Division of Molecular Modification and Cancer Biology, National Cancer Center Research Institute, 5-1-1, Tsukiji, Chuo-ku, Tokyo 104-0045, Japan. Tel: +81 335422511, Fax: +81 335453567, e-mail: kazumkob@ncc.go.jp,

Key Words: Cervical cancer, oligo-recurrence, salvage treatment, radiotherapy.
Federation of Gynecology and Obstetrics (FIGO) stage IIIB cervical cancer treated by definitive radiotherapy (1-3). Once any kind of relapse occurred after definitive radiotherapy, the prognosis may be severely impaired, and the reported 5-year overall survival (OS) rate was only about $10 \%$ (4). This is mainly because the indication of salvage therapy such as individualized radiotherapy or surgical resection became limited because of prior pelvic irradiation, which already had significantly affected sensitive pelvic organs. Thus, careful treatment selection based on risk assessment is necessary for patients with recurrent disease inside or outside of the prior radiation fields.

Oligo-recurrence or oligometastases is generally defined by one to five metastatic lesions and hypothesized to be a limited stage of cancer spread (5). The clinical implication of oligo-recurrence is potentially curable with a localized form of salvage treatment. While emerging data suggest that radiotherapy could play a critical role in local control of the oligo-recurrent state in certain kinds of cancers (6-8), but the evidence regarding cervical cancer is still limited $(9,10)$. Some studies have demonstrated the effectiveness of concurrent chemoradiotherapy as a salvage treatment for patients with lymph node recurrence after radical surgery $(11,12)$. However, the ratio of recurrence is generally higher in the population with locally advanced stages which are usually treated by definitive radiotherapy with or without chemotherapy, but the clinical decision at the time of recurrence can be more difficult because of the impact of prior radiation (13). Thus, to investigate the modality of additional treatment of patients with prior pelvic irradiation, it is essential to identify a population which is expected to benefit in terms of prolonged survival.

In the present study, we compared the clinical outcomes of patients treated by local radiotherapy or systemic chemotherapy for oligo-recurrent diseases with prior pelvic irradiation. The pattern of recurrence by the treatment 
modality was classified to reveal the basis of the clinical decision at the time of recurrence. Furthermore, the prognostic impact of various factors including age, time to recurrence, control of the primary site, treatment modality, number of recurrent sites and pattern of recurrent regions were evaluated for identifying the potentially curable population with oligo-recurrent cervical cancer.

\section{Patients and Methods}

Patient characteristics. Between January 2001 and December 2009, 341 consecutive patients with uterine cervical cancer in FIGO IB to IIIB stages underwent definitive therapy, which included either radical hysterectomy followed by postoperative radiotherapy or definitive radiotherapy with or without chemotherapy, in the National Cancer Center Hospital in Japan. Among them, 98 patients experienced any form of treatment failure by the end of April 2012, which included 41 cases with a solitary or oligo-recurrent ( $\leq 5$ lesions) relapses treated by local radiotherapy (local radiotherapy group; $n=22$ ) or systemic chemotherapy (systemic chemotherapy group; $n=19$ ). The rest of patients $(n=57)$ were cases with multiple ( $>5$ lesions) metastasis, residual tumors from the initial therapy, or any form of recurrent disease on the best supportive care. The oligo-recurrent state was defined just by a number of recurrent sites not greater than five despite whether the primary site was involved or not. All diseases were diagnosed by imaging techniques such as computed tomography (CT), positron emission tomography with $\left[{ }^{18} \mathrm{~F}\right]-$ fluorodeoxyglucose (FDGPET), or magnetic resonance imaging (MRI).

Definitive treatment for cervical cancer. The treatment plan for each patient with gynecological cancer was decided during institutional cancer board meetings. Our standard treatment policy of definitive therapy for newly diagnosed uterine cervical cancer has been described in detail elsewhere $(1,14)$. In short, radical hysterectomy was performed in patients with FIGO IB-IIB stages, while definitive radiotherapy with or without chemotherapy was delivered in patients with FIGO IIIA-IIIB stages.

The medical records of the 41 patients with a solitary or oligorecurrent relapses treated by local radiotherapy (local radiotherapy group; $n=22$ ) or systemic chemotherapy (systemic chemotherapy group; $n=19$ ) were retrospectively reviewed. As an initial treatment, ten patients in FIGO IB-IIB stages underwent radical hysterectomy combined with adjuvant radiotherapy, three patients in FIGO IB-IIB stages, and 28 patients in FIGO IIIA-IIIB stages received definitive radiotherapy with or without concurrent chemotherapy. A combination of external beam radiotherapy with a dose of 40-60 Gy in 20-30 fractions plus intracavitary brachytherapy of 12-24 Gy in 2-4 fractions were the standard course of definitive radiotherapy in our hospital. Most patients were treated by concurrent chemotherapy regimen with cisplatin ( $40 \mathrm{mg} / \mathrm{m}^{2} /$ week); however, some were treated by radiation alone because of advanced age, extensive radiation field including para-aortic lymph region or compromised renal function. Supportive interventions such as blood transfusions were encouraged during radiotherapy.

Treatment for oligo-recurrent cervical cancer. For oligo-recurrent cervical cancer in patients with prior pelvic irradiation, the choice of additional treatment depends on various factors, such as location and distribution of disease, relationship with prior radiation fields, organ function, and performance status. The most emphasized criteria may be the spatial relationship between recurrent lesions and prior radiation fields. Therefore, for patients with good general condition, out-field recurrence is preferentially treated by local radiotherapy, and in-field recurrence was considered as an indication of systemic chemotherapy because of the tolerance of dose of organs at risk.

Details on the treatment for oligo-recurrent cervical cancer are as follows. In the group of local radiotherapy $(n=22)$, conventional three-dimensional technique with a median dose of $50 \mathrm{~Gy}$ (range $=40-75 \mathrm{~Gy}$ ) was used for 21 patients, and intracavitary brachytherapy with prescribed dose of $18 \mathrm{~Gy}$ was applied to only one patient with recurrence in vaginal introitus. The minimum prescribed dose of 40 Gy was still based on the curative intent for the marginal recurrence of prior radiation fields. The other 20 patients received at least a dose of $50 \mathrm{~Gy}$. There was no case of insufficient radiation fields, which did not cover all recurrent sites. No patient received concurrent chemoradiotherapy. In the group of systemic chemotherapy $(\mathrm{n}=19)$, various chemotherapy regimens were used (docetaxel plus cisplatin, 8; TS-1, 3; cisplatin, 2; carboplatin plus CPT-11, 2; docetaxel plus cyclophosphamide, 2; cisplatin plus TS-1, 1; paclitaxel plus carboplatin, 1) based on the individual history of chemotherapy or tolerability. Hereafter, "additional treatment" refers to local radiotherapy or systemic chemotherapy for oligo-recurrent cervical cancer.

Follow-up. All patients were evaluated weekly during additional treatment through physical examination and blood test. CT and/or MRI scans and cytology were performed 1-3 months after additional treatment. Physical examination and blood tests were performed regularly every 1-6 months. Disease progression was defined by the response evaluation criteria in solid tumors (RECIST) version 1.1.

Statistical analysis. Data are presented as mean \pm standard deviation unless otherwise specified. Statistical analysis was performed with JMP version 10.0 (SAS Institute, Cary, North Carolina). Overall survival (OS) was defined as the time between the start of additional treatment and all-cause death or last follow-up. Local recurrencefree survival (LRFS) was estimated from the beginning of additional treatment to the date of local relapse inside the target sites. Distantfree survival (DFS) was estimated from the beginning of additional treatment to the date of distant metastasis out of the target sites. OS, LRFS, and DFS were calculated by the Kaplan-Meier method. Using the log-rank test and generalized Wilcoxon test, the prognostic difference between patients treated with local radiotherapy and systemic chemotherapy was analyzed. Cox hazard proportion model was used in univariate and multivariate analysis for identifying risk factors such as age, time to recurrence, control of primary sites, modality of additional treatment, number of recurrent sites and pattern of recurrent regions. Statistical significance was set at a two-sided $p$-value of less than 0.05 .

\section{Results}

Table I shows the demographic and clinical features of patients. Between the two groups (i.e., local radiotherapy and systemic chemotherapy), a greater number of regions of recurrent sites was observed in patients treated with systemic chemotherapy $(p=0.0004)$. The number of occurrences in 
Table I. Demographic and clinical features of patients.

\begin{tabular}{|c|c|c|c|c|c|c|c|}
\hline & \multicolumn{2}{|c|}{ All patients } & \multicolumn{2}{|c|}{$\begin{array}{l}\text { Patients with local } \\
\text { radiotherapy }\end{array}$} & \multicolumn{2}{|c|}{$\begin{array}{l}\text { Patients with systemic } \\
\text { chemotherapy }\end{array}$} & $p$-Value \\
\hline \multirow{7}{*}{$\begin{array}{l}\text { Number of cases } \\
\text { Age at initial diagnosis (years) } \\
\text { FIGO stage at initial diagnosis }\end{array}$} & \multicolumn{2}{|c|}{41} & \multicolumn{2}{|c|}{22} & \multicolumn{2}{|c|}{19} & \\
\hline & \multicolumn{2}{|c|}{$53.4 \pm 10.4$} & \multicolumn{2}{|c|}{$55.6 \pm 9.3$} & \multicolumn{2}{|c|}{$50.9 \pm 11.2$} & 0.15 \\
\hline & IB & $6(14.6 \%)$ & IB & $3(13.6 \%)$ & IB & $3(15.7 \%)$ & 0.22 \\
\hline & IIA & $1(2.4 \%)$ & IIA & $1(4.5 \%)$ & IIA & $0(0.0 \%)$ & \\
\hline & IIB & $6(14.6 \%)$ & IIB & $1(4.5 \%)$ & IIB & $5(26.3 \%)$ & \\
\hline & IIIA & $3(7.3 \%)$ & IIIA & $3(13.6 \%)$ & IIIA & $0(0.0 \%)$ & \\
\hline & IIIB & $25(60.9 \%)$ & IIIB & $14(63.6 \%)$ & IIIB & $11(57.8 \%)$ & \\
\hline \multirow[t]{2}{*}{ Pathology } & \multirow{2}{*}{\multicolumn{2}{|c|}{$\begin{array}{c}\text { SCC } 37(90.2 \%) \\
\text { Adeno } 4(9.7 \%)\end{array}$}} & \multirow{2}{*}{\multicolumn{2}{|c|}{$\begin{array}{l}\text { SCC } 20(90.9 \%) \\
\text { Adeno } 2(9.0 \%)\end{array}$}} & \multirow{2}{*}{\multicolumn{2}{|c|}{$\begin{array}{l}\text { SCC } 17(89.4 \%) \\
\text { Adeno } 2(10.5 \%)\end{array}$}} & 0.87 \\
\hline & & & & & & & \\
\hline Time to recurrence (months) & \multicolumn{2}{|c|}{$18.4 \pm 17.9$} & \multicolumn{2}{|c|}{$17.6 \pm 15.7$} & \multicolumn{2}{|c|}{$19.4 \pm 20.5$} & 0.75 \\
\hline Number of recurrent sites & \multicolumn{2}{|c|}{$1.5 \pm 1.0$} & \multicolumn{2}{|c|}{$1.2 \pm 0.7$} & \multicolumn{2}{|c|}{$1.8 \pm 1.2$} & 0.05 \\
\hline Number of regions of recurrent sites & \multicolumn{2}{|c|}{$1.3 \pm 0.5$} & \multicolumn{2}{|c|}{$1.0 \pm 0.2 *$} & \multicolumn{2}{|c|}{$1.5 \pm 0.6^{*}$} & $0.004 *$ \\
\hline
\end{tabular}

FIGO: The International Federation of Gynecology and Obstetrics. SCC: squamous cell carcinoma; Adeno: adenocarcinoma; $p$-value was estimated for the statistical difference between the patients with local radiotherapy and systemic chemotherapy; $*$ Statistically significant $(p<0.05)$.

Table II. Number of occurrences in each anatomical region.

\begin{tabular}{|c|c|c|c|c|}
\hline & All patients & $\begin{array}{l}\text { Patients with local } \\
\text { radiotherapy }\end{array}$ & $\begin{array}{l}\text { Patients with systemic } \\
\text { chemotherapy }\end{array}$ & $p$-Value \\
\hline Pelvic lymph region & $7(12.9 \%)$ & $2(9.0 \%)$ & $5(26.3 \%)$ & 0.13 \\
\hline Para-aortic lymph region & $21(22.2 \%)$ & $16(72.7 \%)^{*}$ & $5(26.3 \%)^{*}$ & $0.004 *$ \\
\hline Abdominal lymph region & $2(3.7 \%)$ & $0(0.0 \%)$ & $2(10.5 \%)$ & 0.11 \\
\hline Mediastinal lymph region & $2(3.7 \%)$ & $0(0.0 \%)$ & $2(10.5 \%)$ & 0.11 \\
\hline Supraclavicular lymph region & $2(3.7 \%)$ & $1(4.5 \%)$ & $1(5.2 \%)$ & 0.89 \\
\hline Cervical lymph region & $1(1.8 \%)$ & $0(0.0 \%)$ & $1(5.2 \%)$ & 0.27 \\
\hline Uterine cervical or vaginal region & $12(22.2 \%)$ & $2(9.0 \%)^{*}$ & $10(52.6 \%)^{*}$ & $0.004^{*}$ \\
\hline Liver & $2(3.7 \%)$ & $0(0.0 \%)$ & $2(10.5 \%)$ & 0.11 \\
\hline Lung & $2(3.7 \%)$ & $1(4.5 \%)$ & $1(5.2 \%)$ & 0.89 \\
\hline Bone & $3(5.5 \%)$ & $2(9.0 \%)$ & $1(5.2 \%)$ & 0.67 \\
\hline
\end{tabular}

$p$-Value was estimated for the statistical difference between the patients with local radiotherapy and systemic chemotherapy; *Statistically significant $(p<0.05)$.

each anatomical region is summarized in Table II. The paraaortic region was preferentially treated with radiotherapy $(p=0.004)$, whereas involvement of uterine cervical or vaginal region, which were basically inside or marginal to prior radiation fields, was mainly treated by systemic chemotherapy $(p=0.004)$.

Clinical outcomes of patients were evaluated by the Kaplan-Meier method (Figures 1 and 2). The median followup period after additional treatment was 24.1 months. For all 41 patients, 2-year OS, LRFS, and DFS were $49.9 \%, 64.5 \%$, and $31.6 \%$, respectively. According to the modality of additional treatment, 2-year OS, LRFS, and DFS were $57.0 \%, 86.3 \%$ and $30.0 \%$, respectively, in the group of local radiotherapy, and $41.6 \%, 43.6 \%$ and $36.0 \%$, respectively, in the group of systemic chemotherapy. Compared to systemic chemotherapy, local radiotherapy had significantly superior local control (log-rank test: $p=0.006$, generalized Wilcoxon test: $p=0.01$ ) but showed substantially inferior distant failure (log-rank test: $p=0.34$, generalized Wilcoxon test: $p=0.03$ ) especially in the early follow-up period. The study did not show any statistical difference in OS between the groups (log-rank test: $p=0.48$, generalized Wilcoxon test: $p=0.53$ ).

Next, prognostic factors associated with OS from additional treatment were analyzed (Table III). Univariate analysis demonstrated that initial FIGO stage more than or equal to IIIA was significantly associated with OS [Risk ratio $=2.48$ 
a

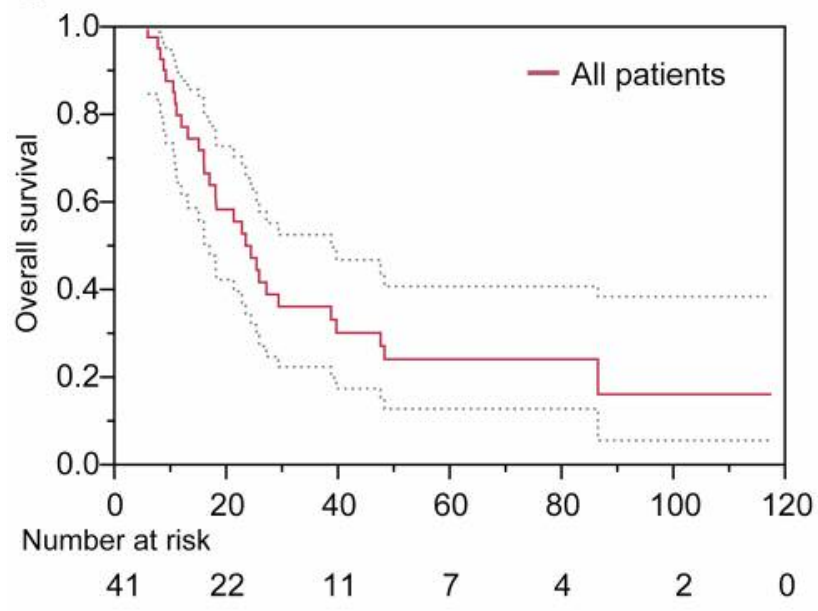

b

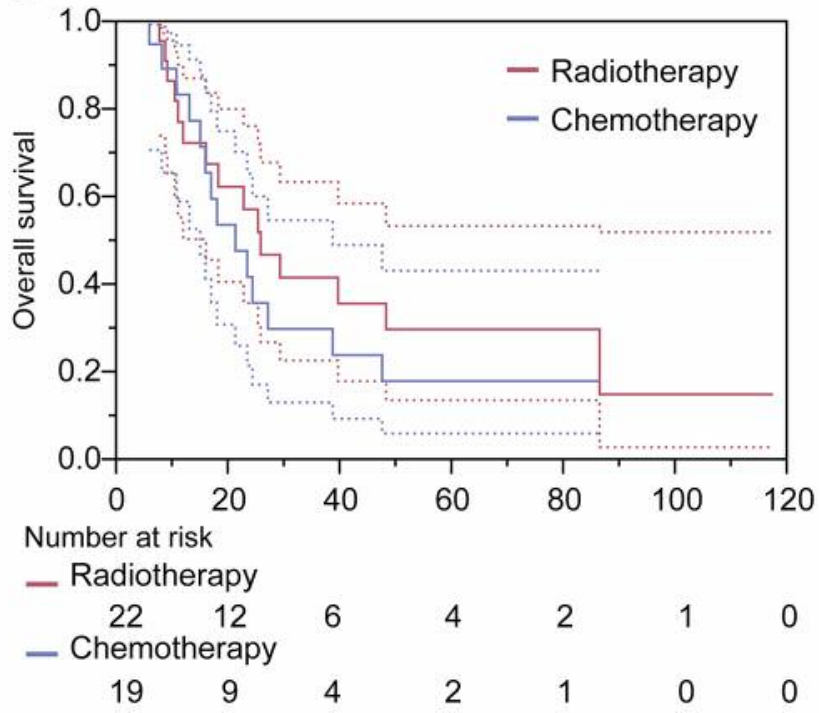

Figure 1. Overall survival calculated by the Kaplan-Meier method. (a) Overall survival of all patients from additional treatment. (b) Comparison of overall survival from additional treatment between patients treated with local radiotherapy and systemic chemotherapy. The log-rank test and generalized Wilcoxon test did not show any significant difference between the groups (log-rank test: $p=0.48$, generalized Wilcoxon test: $p=0.53$ ).

$(95 \% \mathrm{CI}=1.02-7.40) ; p=0.04]$. The other possible factors such as age more than 60 years [Risk ratio $=0.96(95 \% \mathrm{CI}=0.28$ 2.53); $p=0.95]$, time to recurrence more than 10 months [Risk ratio $=0.76(95 \% \mathrm{CI}=0.36-1.66) ; p=0.49]$, control of primary sites (controlled vs. uncontrolled) [Risk ratio $=1.01$ (95\%CI $=0.46-2.45) ; p=0.96]$, treatment modality (local radiotherapy $v s$. systemic chemotherapy) [Risk ratio $=0.76$ (95\% CI $=0.36-1.61) ; p=0.48]$, number of recurrent sites (multiple $v s$. solitary) [Risk ratio $=1.61(95 \% \mathrm{CI}=0.71-3.42)$; $p=0.23$ ] and pattern of recurrent regions [1) only lymph regions $v s$. lymph regions plus central pelvis, 2) only lymph regions vs. lymph regions plus metastasis in organs] [1) Risk ratio, $0.97(95 \% \mathrm{CI}=0.42-2.41) ; p=0.95,2)$ Risk ratio $=0.80$ $(95 \% \mathrm{CI}=0.29-2.83) ; p=0.70]$ were not significantly associated with OS. Initial FIGO stage more than or equal to IIIA still showed clinical significance [Risk ratio=3.12 $(95 \% \mathrm{CI}=1.21$ 9.86); $p=0.01]$ in multivariate analysis following adjustment for the effects of time to recurrence [Risk ratio=0.71 (95\% CI $=0.32-1.59) ; p=0.40]$, treatment modality [Risk ratio $=0.65(95 \% \mathrm{CI}=0.29-1.47) ; p=0.30]$ and number of recurrent sites [Risk ratio $=1.60(95 \% \mathrm{CI}=0.68-3.56) ; p=0.26$ ].

Finally, to reveal the potential subgroup that remains curability by local radiotherapy with oligo-recurrent cervical cancer, te patients were stratified into two groups by FIGO stage. Interestingly, the patients initially diagnosed as FIGO IB-IIB stages treated with local radiotherapy exhibited a trend towards better OS than systemic chemotherapy; however, the effect was not statistically significant (log-rank test: $p=0.07$, generalized Wilcoxon test: $p=0.06)$. On the other hand, patients diagnosed as FIGO IIIA-IIIB stages did not show any difference in OS when treated by local radiotherapy or systemic chemotherapy (log-rank test: $p=0.88$, generalized Wilcoxon test: $p=0.66)$.

\section{Discussion}

Optimal treatment choice for recurrent cervical cancer remains an important clinical question. Careful consideration for the various clinical factors, such as type of initial therapy, the extent of irradiation fields, site of recurrent disease, and patient's general condition, are necessary to be considered in each case (13). Especially, the history of prior radiotherapy to the pelvis has shown to be a negative prognostic factor $(15,16)$, suggesting that it limits the therapeutic window of salvage therapy. In oligo-recurrent cases with prior radiotherapy, carefully selected patients with in-field recurrence can be candidates for salvage radical hysterectomy (17) or pelvic exenteration (18), which has been reported to give patients longer survival benefit rather than systemic chemotherapy or best supportive care (19). However, there are several patients who cannot tolerate intense treatment or whose recurrent sites are beyond the pelvis, making it difficult to perform any salvage surgery. Systemic chemotherapy as an alternative treatment option is not generally considered to provide cure if used as a single therapy (20). Recent advents in chemotherapy proposed 
a

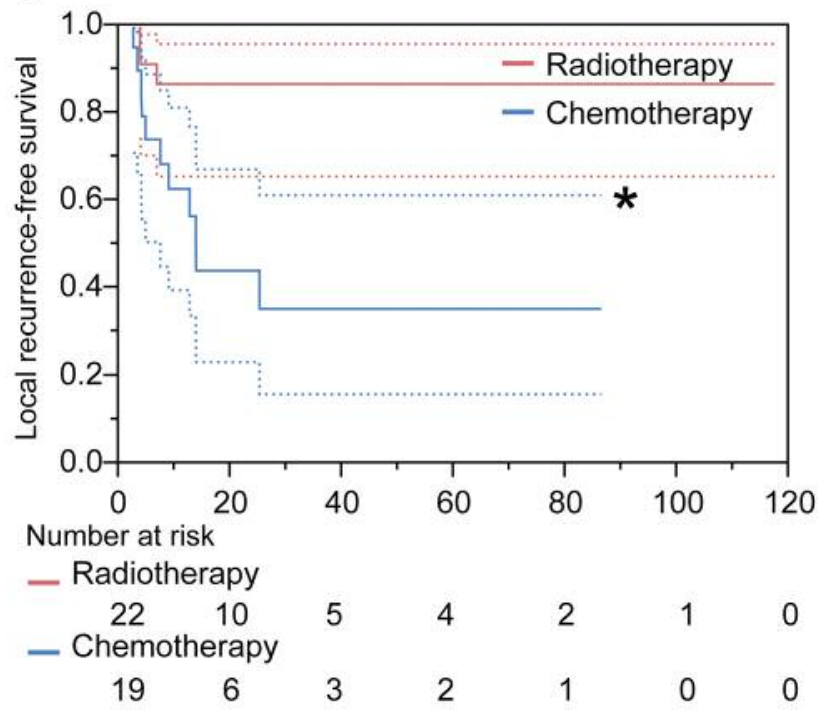

b

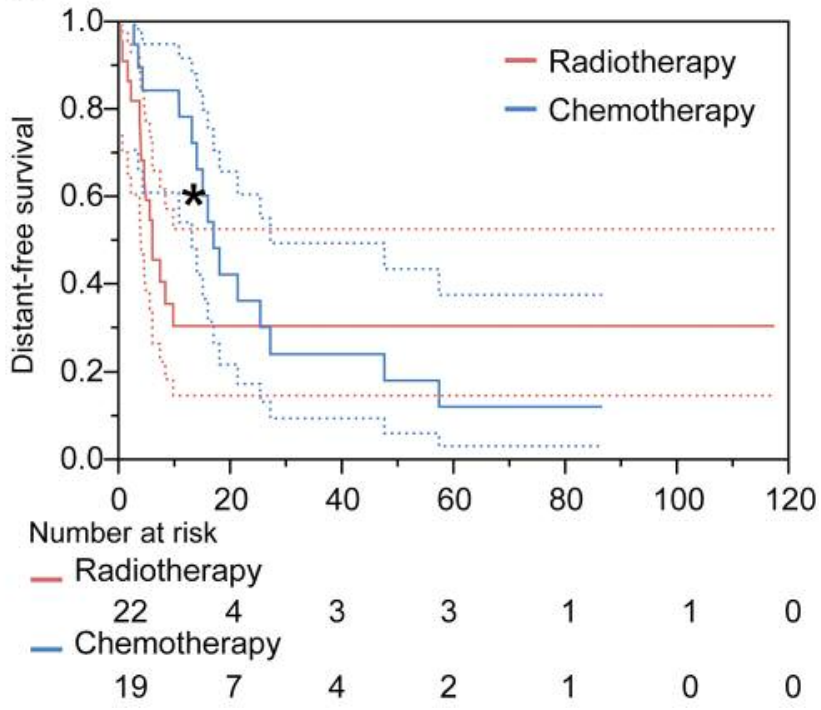

Figure 2. Local recurrence-free survival (LRFS) and distant-free survival (DFS) estimated by the Kaplan-Meier method. (a) LRFS was significantly higher in patients with local radiotherapy than systemic chemotherapy by using the log-rank test (p=0.006). (b) The generalized Wilcoxon test showed that DFS was inferior in patients with local radiotherapy compared to those with systemic chemotherapy (p=0.03). The discrepancy of survival curves can be seen especially at the earlier follow-up period. *Statistically significant $(p<0.05)$.

Table III. Prognostic factors associated with overall survival from additional treatment.

\begin{tabular}{|c|c|c|}
\hline & $\begin{array}{l}\text { Univariate analysis } \\
\text { Risk ratio }(95 \% \mathrm{CI}), p \text {-value }\end{array}$ & $\begin{array}{c}\text { Multivariate analysis } \\
\text { Risk ratio }(95 \% \mathrm{CI}), p \text {-value }\end{array}$ \\
\hline Age at initial diagnosis $>60$ & $0.96(0.28-2.53), 0.95$ & - \\
\hline FIGO stage at initial diagnosis $\geq$ IIIA & $2.48(1.02-7.40), 0.04 *$ & $3.12(1.21-9.86), 0.01 *$ \\
\hline Time to recurrence $>10$ months & $0.76(0.36-1.66), 0.49$ & $0.71(0.32-1.59), 0.40$ \\
\hline Control of primary site (Controlled vs. Uncontrolled) & $1.01(0.46-2.45), 0.96$ & - \\
\hline Treatment modality (Radiotherapy vs. Chemotherapy) & $0.76(0.36-1.61), 0.48$ & $0.65(0.29-1.47), 0.30$ \\
\hline Number of recurrent sites (Multiple $v s$. Solitary) & $1.61(0.71-3.42), 0.23$ & $1.60(0.68-3.56), 0.26$ \\
\hline Pattern of failure [Only lymph nodes vs. (1) & (1) $0.97(0.42-2.41), 0.95$ & - \\
\hline includes central pelvis (2) includes organs] & (2) $0.80(0.29-2.83), 0.70$ & \\
\hline
\end{tabular}

CI: Confidence interval; FIGO: the International Federation of Gynecology and Obstetrics; $p$-value was estimated for the statistical difference between the patients with local radiotherapy and systemic chemotherapy; *Statistically significant $(p<0.05)$.

several agents such as docetaxel, TS-1, carboplatin, CPT-11, cyclophosphamide; however, the common agent in use remains cisplatin (15). Besides, there exists no widely acclaimed standard regimen in the case of recurrent disease with prior radiotherapy despite several clinical trials $(21,22)$. Therefore, we attempted to find a valid indication for local radiotherapy for recurrent cervical cancer especially in the cases with prior pelvic irradiation.

In the present study, local radiotherapy for the oligorecurrent relapse was mostly performed in cases of involvement of para-aortic lymph region (Table II).
Compared to systemic chemotherapy, local radiotherapy was associated with better local control and worse distant failure (Figure 2). Since distant failure may impair OS because of disease progression, attention should be paid for potential spread of cancer beyond the gross tumor sites with modern imaging techniques such as CT or PET (23). Since several studies have implied superior benefit of concurrent chemoradiotherapy to radiotherapy alone for the recurrence after radical hysterectomy $(11,24,25)$, chemotherapy can be added complementary to control the microscopic disease in high-risk cases. From this viewpoint, the significant 
a

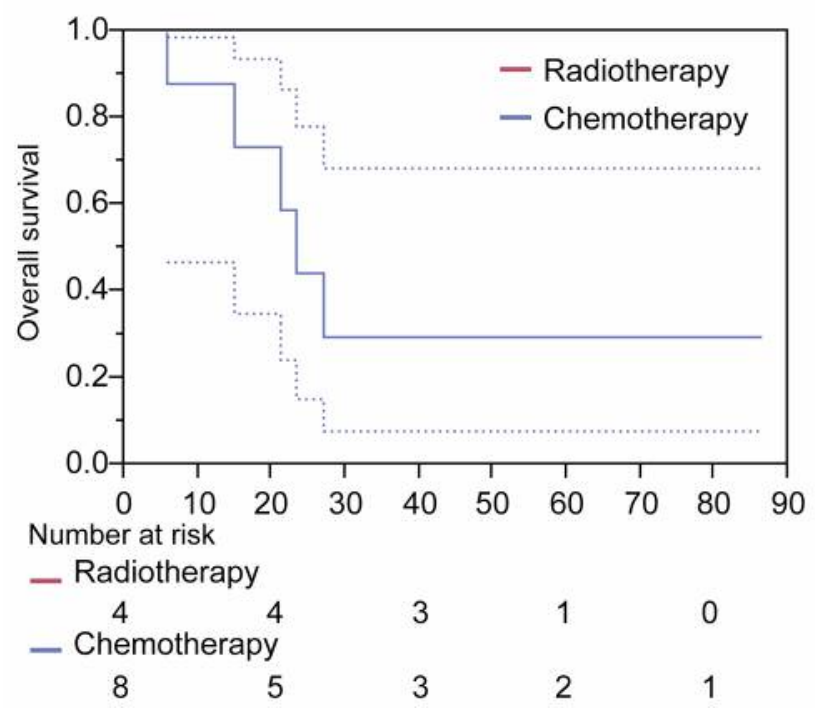

b

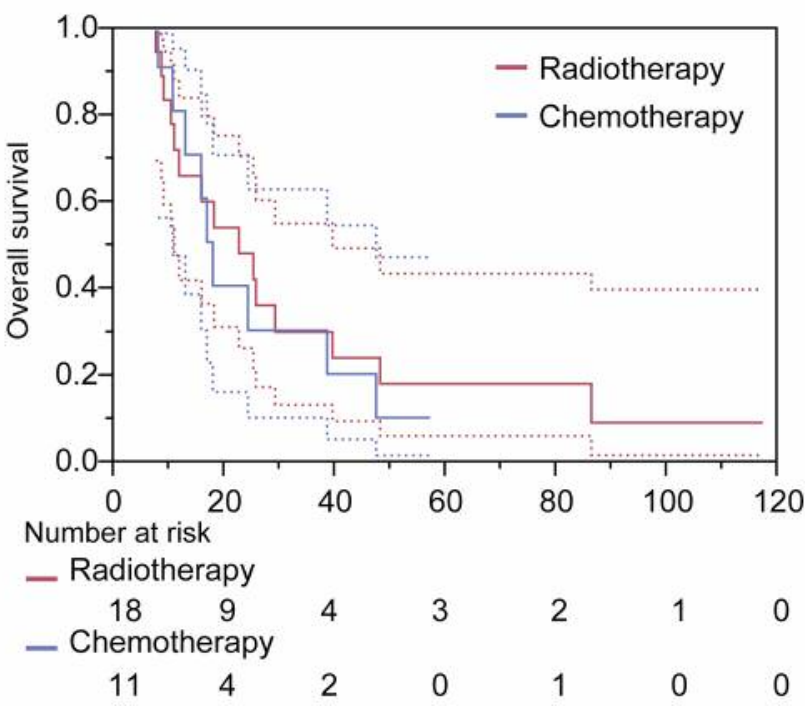

Figure 3. Overall survival curves in patients with local radiotherapy and systemic chemotherapy stratified by FIGO stage. (a) In FIGO IB-IIB stages, patients treated with local radiotherapy showed a trend toward improved overall survival using the log-rank test ( $p=0.07)$. (b) In FIGO IIIA-IIIB stages, there was no substantial difference in overall survival between the groups using the log-rank test $(p=0.88)$.

association between the greater number of recurrent regions and systemic chemotherapy (Table I) seems reasonable to guide clinical decision. However, it is interesting that multivariate analysis found that initial advanced stage but not the number of recurrent sites is a negative factor for OS (Table III). The tendency of better OS in patients initially diagnosed with FIGO IB-IIB stages who were treated with local radiotherapy is remarkable (Figure 3), suggesting that out-field oligo-recurrence of early-stage cervical cancer may be a potentially curable population for whom local radiotherapy can be considered.

There are several limitations to the present study. The retrospective chart review may allow some selection bias of patients and treatment modalities. The generalization to other races, ethnicities and age groups is limited. The relatively small number of patients makes interpretation of statistically non-significant findings challenging. Further investigation will be required to stratify risk groups of oligo-recurrent cervical cancer.

\section{Conflicts of Interest}

The Authors have no conflict of interest to declare.

\section{Authors' Contributions}

All Authors make substantial contributions to conception and design, and/or acquisition of data, and/or analysis and interpretation of data: K.K., N.M., K.T. and K.I. collected cases. K.K. completed all data.
K.K. and N.M. designed the study and analyzed data. K.K., N.M., and J.I. co-wrote the paper. R.H. and J.I. revised critically the article.

\section{Acknowledgements}

This work has been financially supported by Grants in-Aid for Young Scientists (B) from the Ministry of Education, Culture, Sports, Science, and Technology of Japan (Grant number 17K16497).

\section{References}

1 Kuroda Y, Murakami N, Morota M, Sekii S, Takahashi K, Inaba K, Mayahara H, Ito Y, Yoshimura R-I, Sumi M, Kagami Y, Katsumata N, Kasamatsu T and Itami J: Impact of concurrent chemotherapy on definitive radiotherapy for women with FIGO IIIb cervical cancer. J Radiat Res 53: 588-593, 2012. PMID: 22843624. DOI: $10.1093 / \mathrm{jrr} / \mathrm{rrs} 010$

2 Yalman D, Aras AB, Ozkök S, Duransoy A, Celik OK, Ozsaran $\mathrm{Z}$ and Haydaroğlu A: Prognostic factors in definitive radiotherapy of uterine cervical cancer. Eur J Gynaecol Oncol 24: 309-314, 2003. PMID: 12807246.

3 Perez CA, Grigsby PW, Nene SM, Camel HM, Galakatos A, Kao MS and Lockett MA: Effect of tumor size on the prognosis of carcinoma of the uterine cervix treated with irradiation alone. Cancer 69: 2796-2806, 1992. PMID: 12807246.

4 Hong J-H, Tsai C-S, Lai C-H, Chang T-C, Wang C-C, Chou H$\mathrm{H}$, Lee SP and Hsueh S: Recurrent squamous cell carcinoma of cervix after definitive radiotherapy. Int J Radiat Oncol 60: 249257, 2004. PMID: 15337563. DOI: 10.1016/j.ijrobp.2004.02.044

5 Niibe $\mathrm{Y}$ and Hayakawa K: Oligometastases and oligorecurrence: the new era of cancer therapy. Jpn J Clin Oncol 40: 107-111, 2010. PMID: 20047860. DOI: 10.1093/jjco/hyp167 
6 Lo SS, Moffatt-Bruce SD, Dawson LA, Schwarz RE, Teh BS, Mayr NA, Lu JJ, Grecula JC, Olencki TE and Timmerman RD: The role of local therapy in the management of lung and liver oligometastases. Nat Rev Clin Oncol 8: 405-416, 2011. PMID: 21606970. DOI: 10.1038/nrclinonc.2011.75

7 Tree AC, Khoo VS, Eeles RA, Ahmed M, Dearnaley DP, Hawkins MA, Huddart RA, Nutting CM, Ostler PJ and van As NJ: Stereotactic body radiotherapy for oligometastases. Lancet Oncol 14: e28-37, 2013. PMID: 23276369. DOI: 10.1016/S14 70-2045(12)70510-7

8 Ning MS, Gomez DR, Heymach JV and Swisher SG: Stereotactic ablative body radiation for oligometastatic and oligoprogressive disease. Transl Lung Cancer Res 8: 97-106, 2018. DOI: $10.21037 /$ tlcr.2018.09.21

9 Ning MS, Ahobila V, Jhingran A, Stecklein SR, Frumovitz M, Schmeler KM, Eifel PJ and Klopp AH: Outcomes and patterns of relapse after definitive radiation therapy for oligometastatic cervical cancer. Gynecol Oncol 148: 132-138, 2018. PMID: 29089122. DOI: 10.1016/j.ygyno.2017.10.017

10 Hong JH, Tsai CS, Lai CH, Chang TC, Wang CC, Chou HH, Lee SP and Hsueh S: Recurrent squamous cell carcinoma of cervix after definitive radiotherapy. Int J Radiat Oncol Biol Phys 60: 249257, 2004. PMID: 15337563. DOI: 10.1016/j.ijrobp.2004. 02.044

11 Jeon W, Koh HK, Kim HJ, Wu HG, Kim JH and Chung HH: Salvage radiotherapy for lymph node recurrence after radical surgery in cervical cancer. J Gynecol Oncol 23: 168-174, 2012 PMID: 22808359. DOI: 10.3802/jgo.2012.23.3.168

12 Yin Y, Li H, Sheng X, Du X, Wang C, Lu C and Pan C: The treatment of pelvic locoregional recurrence of cervical cancer after radical surgery with intensity-modulated radiation therapy compared with conventional radiotherapy: a retrospective study. Int J Gynecol Cancer 25: 1058-1065, 2015. PMID: 25647254. DOI: $10.1097 /$ IGC.0000000000000360

13 Cibula D, Pötter R, Planchamp F, Avall-Lundqvist E, Fischerova D, Haie Meder C, Köhler C, Landoni F, Lax S, Lindegaard JC, Mahantshetty U, Mathevet P, McCluggage WG, McCormack M, Naik R, Nout R, Pignata S, Ponce J, Querleu D, Raspagliesi F, Rodolakis A, Tamussino K, Wimberger P and Raspollini MR: The European Society of Gynaecological Oncology/European Society for Radiotherapy and Oncology/European Society of Pathology guidelines for the management of patients with cervical cancer. Radiother Oncol 127: 404-416, 2018. PMID: 29728273. DOI: 10.1016/j.radonc.2018.03.003

14 Kasamatsu T, Onda T, Sawada M, Kato T and Ikeda S: Radical hysterectomy for FIGO stage IIB cervical cancer: clinicopathological characteristics and prognostic evaluation. Gynecol Oncol 114: 69-74, 2009. PMID: 19398126. DOI: 10.1016/j.ygyno. 2009.03.026

15 Peiretti M, Zapardiel I, Zanagnolo V, Landoni F, Morrow CP and Maggioni A: Management of recurrent cervical cancer: a review of the literature. Surg Oncol 21: e59-66, 2012. PMID: 22244884. DOI: 10.1016/j.suronc.2011.12.008

16 Seebacher V, Sturdza A, Bergmeister B, Polterauer S, Grimm C, Reinthaller A, Hilal Z and Aust S: Factors associated with postrelapse survival in patients with recurrent cervical cancer: the value of the inflammation-based Glasgow Prognostic Score. Arch Gynecol Obstet 299: 1055-1062, 2019. PMID: 30535923. DOI: $10.1007 / \mathrm{s} 00404-018-4993-0$
17 Boers A, Arts HJG, Klip H, Nijhuis ER, Pras E, Hollema H, Wisman GBA, Nijman HW, Mourits MJE, Reyners AKL, de Bock GH, Thomas G and van der Zee AGJ: Radical Surgery in Patients With Residual Disease After (Chemo)Radiation for Cervical Cancer. Int J Gynecol Cancer 24: 1276-1285, 2014. PMID: 24987914. DOI: 10.1097/IGC.0000000000000171

18 Fotopoulou C, Neumann U, Kraetschell R, Schefold JC, Weidemann H, Lichtenegger $\mathrm{W}$ and Sehouli J: Long-term clinical outcome of pelvic exenteration in patients with advanced gynecological malignancies. J Surg Oncol 101: 507-512, 2010. PMID: 20401918. DOI: 10.1002/jso.21518

19 Ferrandina G, Margariti PA, Smaniotto D, Petrillo M, Salerno MG, Fagotti A, Macchia G, Morganti AG, Cellini N and Scambia G: Long-term analysis of clinical outcome and complications in locally advanced cervical cancer patients administered concomitant chemoradiation followed by radical surgery. Gynecol Oncol 119: 404-410, 2010. PMID: 20817228. DOI: $10.1016 /$ j.ygyno.2010.08.004

20 Serkies K and Jassem J: Systemic therapy for cervical carcinoma current status. Chinese J Cancer Res 30: 209-221, 2018. PMID: 29861606. DOI: 10.21147/j.issn.1000-9604.2018.02.04

21 Moore DH, Blessing JA, McQuellon RP, Thaler HT, Cella D, Benda J, Miller DS, Olt G, King S, Boggess JF and Rocereto TF: Phase III study of cisplatin with or without paclitaxel in stage IVB, recurrent, or persistent squamous cell carcinoma of the cervix: a gynecologic oncology group study. J Clin Oncol 22: 3113-3119, 2004. PMID: 15284262. DOI: 10.1200/JCO. 2004.04.170

22 Ohara T, Kobayashi Y, Yoshida A, Yoshioka N, Yahagi N, Kondo $\mathrm{H}$, Tozawa A, Kiguchi $\mathrm{K}$ and Suzuki N: Combination of irinotecan (CPT-11) and nedaplatin (NDP) for recurrent patients with uterine cervical cancer. Int J Clin Oncol 18: 1102-1106, 2013. PMID: 23095879. DOI: 10.1007/s10147-012-0487-4

23 Chu Y, Zheng A, Wang F, Lin W, Yang X, Han L, Chen Y and Bai L: Diagnostic value of 18F-FDG-PET or PET-CT in recurrent cervical cancer: a systematic review and meta-analysis. Nucl Med Commun 35: 144-150, 2014. PMID: 24177043. DOI: 10.1097/MNM.0000000000000026

24 Cerrotta A, Gardan G, Cavina R, Raspagliesi F, Stefanon B, Garassino I, Musumeci R, Tana S and De Palo G: Concurrent radiotherapy and weekly paclitaxel for locally advanced or recurrent squamous cell carcinoma of the uterine cervix. A pilot study with intensification of dose. Eur J Gynaecol Oncol 23: 115-119, 2002. PMID: 12013105

25 Windschall A, Ott OJ Sauer R and Strnad V: Radiation therapy and simultaneous chemotherapy for recurrent cervical carcinoma. Strahlentherapie und Onkol Organ der Dtsch Röntgengesellschaft. [et al] 181: 545-550, 2005. PMID: 16044224. DOI: $10.1007 / \mathrm{s} 00066-005-1340-8$ 